\title{
Administration of inhalation anesthetics \\ to small animals: Some problems \\ and solutions*
}

\author{
MERRILL F. ELIAS \\ Center for the Study of Aging and Human Development \\ Duke University Medical Center, Durham, North Carolina 27706 \\ G. DOUGLAS BLENKARN \\ Department of Anesthesiology \\ University of North Carolina, Chapel Hill, North Carolina 27514 \\ SCOTT J. SIMMERMAN† \\ Allegheny College, Meadville, Pennsylvania 16335 \\ and \\ GAIL R. MARSH \\ Center for the Study of Aging and Human Development \\ Duke University Medical Center, Durham, North Carolina 27706
}

Some problems inherent in administering inhalation anesthetics to small laboratory animals in studies of memory consolidation are discussed. A simple and inexpensive apparatus which has been designed to eliminate some of these problems is described.

The consolidation theory of memory suggests that a neurophysiological process maintains habit acquisition from trial to trial by fixing the practice effect of a trial after it is over. Among other anesthetics, diethyl ether and halothane (Fluothane) have been administered at varying intervals after a learning trial to determine the point at which central nervous system (CNS) depressant effects of anesthesia will interfere with this consolidation process (e.g., Elias \& Simmerman, 1971; Essman \& Jarvik, 1961; Wimer, 1968). Generally, rats or mice have been anesthetized in glass beakers or jars containing a sponge or cotton soaked with the liquid anesthetic agent.

There are a number of problems inherent in the administration of anesthetics in this manner. Generally, they are related to the vaporization of anesthetic agents (Adriani, 1962). Very high partial pressures of anesthetics are usually created in the anesthetizing chamber. The sudden inhalation of high concentrations of most volatile agents is irritating to the upper respiratory tract. Respiratory irritation may lead to induction of anesthesia characterized by coughing, bronchial spasm, excessive respiratory tract secretions, excitement, and

*Supported by the United Health Services of North Carolina and by Research Training Grant 5T01HD00164 from the National Institute of Child Health and Human Development. Appreciation is extended to Dr. Joseph C. Farmer, Jr., Department of Surgery, Duke University Medical Center, for his helpful suggestions.

+ Now a graduate student at Iowa State University. concomitant sympatho-adrenal depth is associated with severe depression of the respiratory and cardiovascular systems so that hypoxia and acidosis may be superimposed upon general anesthesia. Moreover, when air in the anesthetizing chamber is used as the carrier gas for the anesthetic vapor, further derangement of respiratory gas exchange occurs as high partial pressure of the anesthetic agent encroaches upon the fractional concentration of oxygen in the air. This hypoxia-creating effect is more pronounced toward the bottom of the chamber, due to the stratification of the denser anesthetic vapor. In addition, the utilization of oxygen and excretion of carbon dioxide within a small closed chamber further reduces the partial pressure of oxygen in the respiratory environment.

Another major objection to anesthetizing small animals within closed unventilated containers is the unpredictable and extremely variable vapor tensions of anesthetics produced. As volatile anesthetics are vaporized, a large amount of heat is extracted from the immediate vaporization). This phenomenon results in undesirable cooling of the chamber and of the experimental animal. The drop in temperature reduces the rate of vaporization and thereby leads to a variable and everchanging concentration of anesthetic vapor in the respiratory environment.

Cherkin (1968), who has discussed the relationship of temperature to the dose and duration of amnesic agents, overactivity. Excessive anesthetic environment (the latent heat of has cited instances of excessive mortality with the "ether jar procedure" when exposure to anesthesia was prolonged over $2 \mathrm{~min}$. The existence of any anesthetic mortality or significant weight loss in experimental animals is sufficient to indict the anesthetic technique. Further, it casts serious doubts upon interpretations of retrograde amnesia in terms of anesthetic effects on electrochemical activity per se because effects of brain damage or general deficit in health are not precluded.

Apparatus used in clinical anesthesia will eliminate these undesirable consequences. A list of companies which offer such apparatus can be found in a publication by Ayerst Laboratories (1967). These instruments are costly and investigators may wish to use less expensive apparatus which can be constructed of materials available in most laboratories. Figure 1 shows a schematic diagram of a simple and inexpensive apparatus that has been used in our laboratory to administer anesthesia to mice in a safe, reliable, and quantifiable manner.

Air is metered through a flow meter and delivered to a stoppered pint bottle containing liquid anesthetic (halothane). Air circulating through the pint bottle conducts anesthetic vapor to the chamber. The bottle is surrounded by a water jacket, and thermometers monitor the temperatures of both the liquid anesthetic and the surrounding water. During the initial period of utilization, the percentage concentration of anesthetic falls. This is due to the drop in temperature of the liquid halothane and $a$ reduction in the rate of concomitant escape of molecules from the liquid into the vapor state. The water jacket helps to stabilize the temperature of the anesthetic liquid and thereby helps to stabilize the rate of anesthetic vaporization and delivery. After a short initial period of equilibration (e.g., $15 \mathrm{~min}$ ), a relatively constant percentage of halothane is delivered to the anesthetizing chamber.

The chamber is constructed of clear Plexiglas and rendered airtight by applying ethylene dichloride to the joints and the tubing connections. 
THERMOMETERS

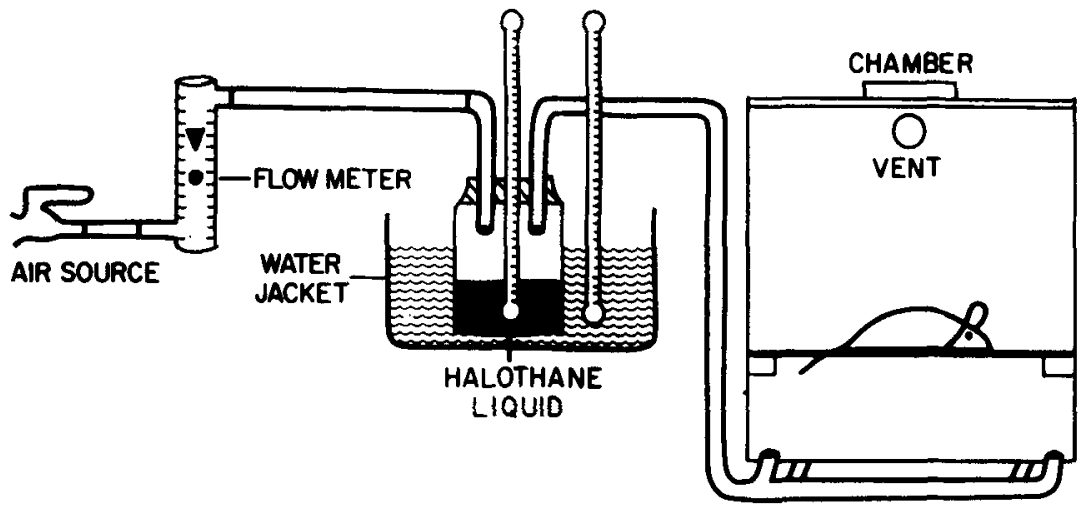

Fig. 1. A simple and inexpensive apparatus for the administration of inhalation anesthetics to small animals.

Animals are placed on a wire mesh platform $5 \mathrm{~cm}$ above the four tubes which bring the anesthetic vapor into the base of the chamber at its four corners. A tight seal is created between the lid and the body of the chamber by means of a siliconized rubber gasket. Tubing connected to a vent (12 mm diam) at the top of the chamber provides an exhaust system which allows airflow and helps to stabilize the pressure in the chamber. If ventilation in the laboratory is poor, a vacuum should be loosely connected to the exhaust tubing. If diethyl ether is used, the exhausted vapor should be bubbled through water to insure that it does not harm vacuum equipment. The percentage of anesthetic delivered to the chamber is regulated by means of adjustments in airflow. At relatively low flow rates, e.g., 1 to 3 liters $/ \mathrm{min}$, an increase in flow rate results in a higher concentration of anesthetic in the chamber atmosphere. The percentage of anesthetic delivered for a given airflow may be determined by means of infrared analyses or gas chromatography. These instruments are usually available in basic science laboratories or in major hospitals which employ general anesthesia. The percentage of anesthetic delivered is determined by inserting a sampling catheter in the line between the bottle containing liquid anesthetic and the chamber. The percentage of anesthetic vapor in the chamber can be determined by inserting a sampling catheter in the exhaust tube at the point where it exits from the chamber.

While we have used this apparatus to study the effect of halothane on retrograde amnesia in mice, it can be used with other inhalation anesthetics and with a variety of small animals in different experiments. It is important to appreciate, however, that the concentration of anesthetic in the chamber is a function of many variables, including airflow, ambient temperature, volume and surface area of both the liquid anesthetic and the water jacket, and the size of the anesthetizing chamber. Having controlled these variables, the investigator can be assured that the delivery of anesthetic will be constant, and that hypoxia, hypercarbia, acidosis, erratic anesthetic concentrations, and other undesirable consequences of poor anesthetic technique will be eliminated.

In an ongoing experiment by the first author, 8-week-old male mice of the BALB/CBy and $\mathrm{C} 57 \mathrm{BL} / 6 \mathrm{By}$ strains provided by Bailey ${ }^{1}$ were anesthetized once a day for 30 consecutive days. The animals were anesthetized immediately after a single discrimination trial and remained in the chamber for $30 \mathrm{sec}$ after the righting reflex was lost. A 3-liter chamber was used, and 1 liter $/ \mathrm{min}$ airflow was metered into a 500-ce glass bottle filled with $50 \mathrm{cc}$ of halothane liquid. The liquid was surrounded by a 3-liter water jacket. After a 15-min period required for equilibration, the temperature of the halothane and the water plateaued at $16^{\circ}$ to $17^{\circ} \mathrm{C}$ and $20^{\circ}$ to $21^{\circ} \mathrm{C}$, respectively. An Instrumentation Associates Narkometer Nack Vonderschmitt MT/672-2 infrared analyzer confirmed delivery of $6 \%$ halothane to the chamber. Opening and closing the chamber to replace animals with 30 to 45 sec between replacement resulted in a variation in anesthetic concentration in the chamber between $5 \%$ and $6 \%$. The mean time from placement in the chamber to loss of righting reflex (induction period) and the mean time to recovery of righting reflex (recovery time) were determined. The BALB/cBy mice showed a mean induction period of $13.72 \mathrm{sec}(\mathrm{SD}=1.79 \mathrm{sec}, \mathrm{N}=18)$ and a mean recovery time of $28.72 \mathrm{sec}$ $(\mathrm{SD}=3.68)$. The $\mathrm{C57BL} / 6 \mathrm{By}$ mice showed a mean induction period of $10.37 \mathrm{sec}(\mathrm{SD}=.82, \quad \mathrm{~N}=16)$ and $a$ mean recovery time of $33.87 \mathrm{sec}$ $(\mathrm{SD}=2.57)$. There was no mortality, and Ss showed a growth and activity pattern which was comparable to control groups. Lowering of the concentration of anesthetic vapor in the chamber would permit the animals to remain in an anesthetized state for a longer period of time than that dictated by the design of the experiment which is in progress.

Regardless of whether investigators elect to use apparatus designed for clinical anesthesia, apparatus similar to that described in this paper, or apparatus dictated by their own ingenuity, it appears to be imperative that the ether jar procedure, with its many inherent problems, be rejected in favor of techniques which permit control over and specification of anesthetic concentrations. Such techniques may help to eliminate the multiple and conflicting interpretations of retrograde amnesia data which appear to characterize this literature.

\section{REFERENCES}

ADRIANI, J. The chemistry and physics of anesthesia. New York: Thomas, 1962.

AYERST LABORATORIES. Fluothane (halothane). For precision inhalation anesthesia. A guide to its use based on a decade of clinical experiment. Ayerst Laboratories, New York, N.Y., 1967.

CHERKIN, A. Retrograde amnesia: Role of temperature, dose, and duration of amnesic agent. Psychonomic Science. $1968,13,255-256$.

ELIAS, M. F., \& SIMMERMAN, S. J. Proactive and retroactive effects of diethvl ether on discrimination learning in $D B A / 2 \mathrm{~J}$ and $C 57 \mathrm{BL} / 6 \mathrm{~J}$ mice. Psy chonomic Science, 1971, 22, 299-301.

ESSMAN, W. B., \& JARVIK, M. E. Impairment of retention for a conditioned response by ether anesthesia in mice. Psychopharmacologica, 1961, 2, $172-179$.

WIMER, B. J. Bases of a facilitative effect upon retention resulting from posttrial etherization. Journal of Comparative \& Physiological Psychology, 1968, 65, 340-342.

\section{NOTE}

1. Thanks are extended to Dr. Donald W. Bailey of The Jackson Laboratory, Bar Harbor, Maine, for supplying animals from his private stocks. 